\author{
United Nations Educational Scientific and Cultural Organization \\ and \\ International Atomic Energy Agency
}

THE ABDUS SALAM INTERNATIONAL CENTRE FOR THEORETICAL PHYSICS

\title{
THE USE OF LORENTZ GROUP FORMALISM IN SOLVING POLARIZATION EFFECTS OF A BIREFRINGENT SINGLE MODE OPTICAL FIBER
}

\author{
G.C. Ishiekwene ${ }^{1}$ \\ Laser and Fiber Optics Center, University of Cape Coast, Cape Coast, Ghana \\ and \\ The Abdus Salam International Centre for Theoretical Physics, Trieste, Italy, \\ C.S. Brown \\ Lucent Technologies, Transmission Media Laboratory, \\ 2000 Northeast Expressway, Norcross, GA 30071, USA, \\ S.Y. Mensah \\ Laser and Fiber Optics Center, University of Cape Coast, Cape Coast, Ghana \\ and \\ A.E. $\mathrm{Bak}^{2}$ \\ Department of Physics, Morehouse College, \\ 830 Westview Drive SW, Atlanta, GA 30314, USA. \\ MIRAMARE - TRIESTE
}

July 2000

\footnotetext{
${ }^{1}$ Regular Associate of the Abdus Salam ICTP.

Permanent address: University of Liberia, Monrovia, Liberia. E-mail: ishiekwene@usa.net

${ }^{2}$ Formerly Fuad Muhammad.
} 


\begin{abstract}
A theoretical analysis on the polarization effects of a light beam propagating in a birefrigent single-mode fiber is presented. We derive a system of differential equations representing the evolution of Stokes parameters and illustrate their application to polarization effects in a straight birefringent single mode optical fiber. The solutions to the set of equations are obtained using specifically the methods of the unified formalism for polarization optics which adopt the use of the Stokes-Mueller equation and the Lorentz group to model polarization phenomena in media such as optical fibers. The analytical results presented using this approach are identical to results obtained from other conventional methods. We observe the characteristic exponential decrease in the total intensity of the input light due to atteuation by the fiber.
\end{abstract}




\section{INTRODUCTION}

The evolution of the state of polarization of a light beam propagating along a birefringent single mode optical fiber can be described using several analytical and graphical methods. One approach involves using a straightforward method of calculating how the electric field varies as the wave propagates along the fiber. In such an approach, an understanding of how the polarization evolves as the beam propagates is not immediately obvious from the complex slowly varying electric field amplitude [1]. The Jones matrix formalism is another approach that has been used to study the rotational effects of polarization in optical fibers [2]. Svirko and Zheludev [3] used tensor analysis to obtain expressions that describe the response of a birefringent fiber to the propagating beam. Several other authors use an alternative method of the dynamical equation for Stokes parameters to describe the polarization changes of a wave propagating in an optical medium [4] and [5]. The use of Stokes parameters has an advantage of providing a rather straightforward analytical definition of different polarization states of light. Daino et al [6] analysed the evolution of the state of polarization along a nonlinear single-mode birefringent fiber using Stokes parameters and gave exact solutions illustrated by means of a Poincaré sphere representation.

In this work, the polarization state of a lightwave is expressed in terms of Stokes parameters. Using the method of ordered exponential operator [7], a formal solution to the system of differential equations is obtained in terms of the Stokes-Mueller equation which has a Mueller matrix containing the information necessary to characterize the fiber so that its effect on the state of polarization can be deduced and the Stokes parameters of the outgoing and incident light beams become known. The elements of the Mueller matrix for different sources of perturbations are then obtained using the Lorentz group formalism.

\section{THEORY}

To analyze the polarization dynamics of a light beam propagating along a birefringent single mode optical fiber, we begin by deriving a system of four coupled differential equations representing the evolution of polarization along the optical fiber. We use the methods of Svirko and Zhedulev [3] to derive the system of coupled differential equations which represent the evolution equations for Stokes parameters for the case in which both linear birefringence and dichroism are present in the fiber and are parallel to one another in Stokes space 


$$
\begin{aligned}
& \frac{d S_{0}}{d z}=-\Delta S_{0}-2 \delta^{\prime \prime} \cos 2 \phi S_{1}-2 \delta^{\prime \prime} \sin 2 \phi S_{2} \\
& \frac{d S_{1}}{d z}=-\Delta S_{1}-2 \delta^{\prime \prime} \cos 2 \phi S_{0}-2 \delta^{\prime} \sin 2 \phi S_{3} \\
& \frac{d S_{2}}{d z}=-\Delta S_{2}-2 \delta^{\prime \prime} \sin 2 \phi S_{0}+2 \delta^{\prime} \cos 2 \phi S_{3} \\
& \frac{d S_{3}}{d z}=-\Delta S_{3}-2 \delta^{\prime} \cos 2 \phi S_{2}+2 \delta^{\prime} \sin 2 \phi S_{1}
\end{aligned}
$$

Eq.(1) can be expressed in matrix form as

$$
\frac{d}{d z}\left(\begin{array}{c}
S_{0} \\
S_{1} \\
S_{2} \\
S_{3}
\end{array}\right)=\left(\begin{array}{cccc}
-\Delta & -2 \delta^{\prime \prime} \cos 2 \phi & -2 \delta^{\prime \prime} \sin 2 \phi & 0 \\
-2 \delta^{\prime \prime} \cos 2 \phi & -\Delta & 0 & -2 \delta^{\prime} \sin 2 \phi \\
-2 \delta^{\prime \prime} \sin 2 \phi & 0 & -\Delta & 2 \delta^{\prime} \cos 2 \phi \\
0 & 2 \delta^{\prime} \sin 2 \phi & -2 \delta^{\prime} \cos 2 \phi & -\Delta
\end{array}\right)\left(\begin{array}{c}
S_{0} \\
S_{1} \\
S_{2} \\
S_{3}
\end{array}\right)
$$

$\Delta$ is the isotropic loss of the fiber, $\delta^{\prime}$ represents anisotropy in the fiber, and $\delta^{\prime \prime}$ relates to its diattenuation effects. $\left\{S_{0}, S_{1}, S_{2}, S_{3},\right\}$ are the Stokes parameters and $z$ is an axis that coincides with the axis of the fiber along which the fields propagate. $\phi$ is an angle between the laboratory and natural coordinate frames of the fiber.

Solutions to the set of equations can be expressed compactly in terms of the Stokes-Mueller matrix equation in the form

$$
\overrightarrow{\mathbf{S}}=\widehat{\mathbf{M}} \cdot \overrightarrow{\mathbf{S}}(0)
$$

where $\widehat{\mathbf{M}}$ is the Mueller matrix of the perturbed single mode fiber. $\overrightarrow{\mathbf{S}}$ and $\overrightarrow{\mathbf{S}}(0)$ represent the Stokes 4-vector notation for the input and output Stokes parameters, respectively.

After expanding in terms of the ordered exponential operators [7], the $4 \times 4$ Mueller matrix $\widehat{\mathbf{M}}$ can be explicitly written for uniform birefringence and dichroism as [8]

$$
\widehat{\mathbf{M}}(\Delta, \mathbf{d}, \boldsymbol{\beta}, z)=\exp (-\Delta z) \exp [(\mathbf{d} \cdot \hat{\mathbf{D}}) z+(\overrightarrow{\boldsymbol{\beta}} \cdot \hat{\mathbf{B}}) z]
$$

where $\mathbf{d}=\left\{\mathbf{d}_{1}, \mathbf{d}_{2}, \mathbf{d}_{3}\right\}$ is the dichroism 3 -vector related to the polarization dependent loss effects of the fiber and $\overrightarrow{\boldsymbol{\beta}}=\left\{\overrightarrow{\boldsymbol{\beta}}_{1}, \overrightarrow{\boldsymbol{\beta}}_{2}, \overrightarrow{\boldsymbol{\beta}}_{3}\right\}$ is the birefringence 3 -vector related to the fiber anisotropy. We note that $\mathbf{d}=\left\{2 \delta^{\prime \prime} \cos 2 \phi, 2 \delta^{\prime \prime} \sin 2 \phi, 0\right\}$ and $\overrightarrow{\boldsymbol{\beta}}=\left\{2 \delta^{\prime} \cos 2 \phi, 2 \delta^{\prime} \sin 2 \phi, 0\right\}$. Also, $\stackrel{\mathbf{D}}{=}=\left\{\hat{\mathbf{D}}_{1}, \hat{\mathbf{D}}_{2}, \hat{\mathbf{D}}_{3}\right\}$ and $\hat{\mathbf{B}}=\left\{\hat{\mathbf{B}}_{1}, \hat{\mathbf{B}}_{2}, \hat{\mathbf{B}}_{3}\right\}$ are six matrices which form an appropriate set of Lorentz generators for dichroism and birefringence, respectively.

The Mueller approach involves the Stokes parameters collectively behaving similar to a Lorentz 4-vector. In this case, the Mueller matrices are essentially four-dimensional Lorentz 
transformations acting on the Stokes 4-vector [9]. A Lorentz transformation is any matrix that can be expressed as [10]

$$
L(\mathbf{v}, \mathbf{u})=\exp [(\mathbf{v} \cdot \mathbf{K})+(\mathbf{u} \cdot \mathbf{J})]
$$

where $\mathbf{v}$ is a real 3-vector (with cartesian components $\mathbf{v}_{1}, \mathbf{v}_{2}, \mathbf{v}_{3}$ ) and $\mathbf{u}$ is a real 3 -vector (with cartesian components $\left.\mathbf{u}_{1}, \mathbf{u}_{2}, \mathbf{u}_{3}\right)$. A transformation for which $\mathbf{u}=0$ is a rotation matrix and a transformation for which $\mathbf{v}=0$ is a boost matrix. The notations $\mathbf{K}$ and $\mathbf{J}$ are each shorthand for three matrices: $\left\{\mathbf{K}_{m}, \mathbf{J}_{m} \quad m=1,2,3\right\}$. These matrices are called generators, and they satisfy the following commutation relations

$$
\begin{gathered}
{\left[\mathbf{J}_{l}, \mathbf{J}_{m}\right]=-\sum_{n=1}^{3} \varepsilon_{l m n} \mathbf{J}_{n}} \\
{\left[\mathbf{K}_{l}, \mathbf{K}_{m}\right]=\sum_{n=1}^{3} \varepsilon_{l m n} \mathbf{J}_{n}} \\
{\left[\mathbf{J}_{l}, \mathbf{K}_{m}\right]=-\sum_{n=1}^{3} \varepsilon_{l m n} \mathbf{K}_{n}}
\end{gathered}
$$

where $\varepsilon_{l m n}$ is component of the three-dimensional totally antisymmetric tensor with $\left(\varepsilon_{123}=+1\right)$.

We note that the matries $\hat{\mathbf{D}}$ and $\hat{\mathbf{B}}$ in Eq.(4) are the generators of the irrep $\left(\frac{1}{2}, \frac{1}{2}\right)$ of the Lorentz group such that

$$
\hat{\mathbf{D}}=\mathbf{K}^{\left(\frac{1}{2}, \frac{1}{2}\right)} \quad, \quad \stackrel{\wedge}{\mathbf{B}}=\mathbf{J}^{\left(\frac{1}{2}, \frac{1}{2}\right)}
$$

where the irrep $\left(\frac{1}{2}, \frac{1}{2}\right)$ is the vector representation of the Lorentz group. It is a four-dimensional real representation with generators

$$
\begin{aligned}
& \mathbf{J}_{1}^{\left(\frac{1}{2}, \frac{1}{2}\right)}=\left(\begin{array}{llll}
0 & 0 & 0 & 0 \\
0 & 0 & 0 & 0 \\
0 & 0 & 0 & 1 \\
0 & 0 & -1 & 0
\end{array}\right)=\hat{\mathbf{B}}_{1} \quad, \quad \mathbf{J}_{2}^{\left(\frac{1}{2}, \frac{1}{2}\right)}=\left(\begin{array}{llll}
0 & 0 & 0 & 0 \\
0 & 0 & 0 & 1 \\
0 & 0 & 0 & 0 \\
0 & -1 & 0 & 0
\end{array}\right)=\hat{\mathbf{B}}_{2} \\
& \mathbf{J}_{3}^{\left(\frac{1}{2}, \frac{1}{2}\right)}=\left(\begin{array}{llll}
0 & 0 & 0 & 0 \\
0 & 0 & 1 & 0 \\
0 & -1 & 0 & 0 \\
0 & 0 & 0 & 0
\end{array}\right)=\hat{\mathbf{B}}_{3} \quad, \quad \mathbf{K}_{1}^{\left(\frac{1}{2}, \frac{1}{2}\right)}=\left(\begin{array}{llll}
0 & -1 & 0 & 0 \\
-1 & 0 & 0 & 0 \\
0 & 0 & 0 & 0 \\
0 & 0 & 0 & 0
\end{array}\right)=\hat{\mathbf{D}}_{1} \\
& \mathbf{K}_{2}^{\left(\frac{1}{2}, \frac{1}{2}\right)}=\left(\begin{array}{llll}
0 & 0 & & 0 \\
0 & 0 & 0 & 0 \\
-1 & 0 & 0 & 0 \\
0 & 0 & 0 & 0
\end{array}\right)=\hat{\mathbf{D}}_{2} \quad, \quad \mathbf{K}_{3}^{\left(\frac{1}{2}, \frac{1}{2}\right)}=\left(\begin{array}{llll}
0 & 0 & 0 & -1 \\
0 & 0 & 0 & 0 \\
0 & 0 & 0 & 1 \\
-1 & 0 & 0 & 0
\end{array}\right)=\hat{\mathbf{D}}_{3}
\end{aligned}
$$


Thus the Mueller matrix of the perturbed single mode fiber is essentially a Lorentz vector transformation. Then, the Mueller matrix for a purely birefringent single mode fiber is a rotation matrix. That is,

$$
\widehat{\mathbf{M}}(0,0, \vec{\beta}, z)=\left[\hat{\mathbf{I}}+\left(\mathbf{e}_{\beta} \cdot \hat{\mathbf{B}}\right)^{2}\right]-\left(\mathbf{e}_{\beta} \cdot \hat{\mathbf{B}}\right)^{2} \cos \beta z+\left(\mathbf{e}_{\beta} \cdot \hat{\mathbf{B}}\right) \sin \beta z
$$

where $\beta=2 \delta^{\prime}$ is the magnitude of $\overrightarrow{\boldsymbol{\beta}}$, and

$$
\mathbf{e}_{\beta}=\frac{\overrightarrow{\boldsymbol{\beta}}}{\beta}=\beta_{1} \mathbf{e}_{1}+\beta_{2} \mathbf{e}_{2}+\beta_{3} \mathbf{e}_{3}
$$

is a unit vector in the direction of the total birefringence.

We now write $\widehat{\mathbf{M}}(0,0, \vec{\beta}, z)$ more explicitly as

$$
\widehat{\mathbf{M}}(0,0, \overrightarrow{\boldsymbol{\beta}}, z)=\left(\begin{array}{cccc}
1 & 0 & 0 & 0 \\
0 & \cos 2 \phi+\sin ^{2} 2 \phi \cos \beta z & \sin 2 \phi \cos 2 \phi(1-\cos \beta z) & -\sin 2 \phi \sin \beta z \\
0 & \sin 2 \phi \cos 2 \phi(1-\cos \beta z) & \sin ^{2} 2 \phi+\cos ^{2} 2 \phi \cos \beta z & \cos 2 \phi \sin \beta z \\
0 & \sin 2 \phi \sin \beta z & -\cos 2 \phi \sin \beta z & \cos \beta z
\end{array}\right)
$$

Also, the Mueller matrix for a purely dichroic single mode fiber is the product of a scalar exponential factor and a boost matrix. Thus,

$$
\widehat{\mathbf{M}}(\Delta, \mathbf{d}, 0, z)=\exp (-\Delta z)\left[\hat{\mathbf{I}}-\left(\mathbf{e}_{d} \cdot \hat{\mathbf{D}}\right)^{2}\right]+\left(\mathbf{e}_{d} \cdot \hat{\mathbf{D}}\right)^{2} \cosh d z+\left(\mathbf{e}_{d} \cdot \hat{\mathbf{D}}\right) \sinh d z
$$

where $d=2 \delta^{\prime \prime}$ is the magnitude of $\mathbf{d}$, and

$$
\mathbf{e}_{d}=\frac{\mathbf{d}}{d}=d_{1} \mathbf{e}_{1}+d_{2} \mathbf{e}_{2}+d_{3} \mathbf{e}_{3}
$$

is a unit vector in the direction of the total dichroism. $\hat{\mathbf{I}}$ is the $4 \times 4$ identity matrix. Similarly, Eq.(12) can be written more explicitly as

$$
\exp (-\Delta z)\left(\begin{array}{cccc}
C_{h} & \widehat{\mathbf{M}}(\Delta, \mathbf{d}, 0, z)= & \\
-\cos 2 \phi \sinh d z & \sin ^{2} 2 \phi+\cos ^{2} 2 \phi \cosh d z & \sin 2 \phi \cos 2 \phi \sinh d z & 0 \\
-\sin 2 \phi \sinh d z & \sin 2 \phi \cos 2 \phi(\cosh d z-1) & \cos ^{2} 2 \phi+\sin ^{2} 2 \phi \cosh d z & 0 \\
0 & 0 & 0 & 1
\end{array}\right)
$$

The Mueller matrices for pure birefringence and dichroism are Lorentz transformations and therefore have specific geometrical interpretations. In the next section, we discuss specific cases of the Stokes-Mueller matrix equation for different input polarization of the propagating light beam. 


\section{RESULTS}

The Mueller matrix which characterizes the perturbations due to the presence of linear birefringence and dichroism in the fiber can therefore be obtained and expressed as the composite of the individual matrices due to only birfringence and only dichroism because the individual Mueller matrices commute. In other words, the product of equations (11) and (14) yields the Mueller matrix for the fiber. Hence, the Stokes Mueller equation can be written as

$$
\left(\begin{array}{c}
S_{0} \\
S_{1} \\
S_{2} \\
S_{3}
\end{array}\right)=e^{-\Delta z}\left(\begin{array}{cccc}
C_{h} & -C_{2 \phi} S_{h} & -S_{2 \phi} S_{h} & 0 \\
-C_{2 \phi} S_{h} & C_{2 \phi}^{2} C_{h}+S_{2 \phi}^{2} C_{\beta} & C_{2 \phi} S_{2 \phi}\left(C_{h}-C_{\beta}\right) & -S_{2 \phi} S_{\beta} \\
-S_{2 \phi} S_{h} & C_{2 \phi} S_{2 \phi}\left(C_{h}-C_{\beta}\right) & S_{2 \phi}^{2} C_{h}+C_{2 \phi}^{2} C_{\beta} & C_{2 \phi} S_{\beta} \\
0 & S_{2 \phi} S_{\beta} & -C_{2 \phi} S_{\beta} & C_{\beta}
\end{array}\right)\left(\begin{array}{c}
S_{00} \\
S_{10} \\
S_{20} \\
S_{30}
\end{array}\right)
$$

where $C_{h}=\cosh d z, S_{h}=\sinh d z, S_{2 \phi}=\sin 2 \phi, C_{2 \phi}=\cos 2 \phi, C_{\beta}=\cos \beta z$ and $S_{\beta}=\sin \beta z$.

The elements of the matrix in Eq.(15) can be expressed in functional forms to obtain the output Stokes parameters for different input polarization of a light beam propagating along a birefringent single mode fiber. Using these functional forms, graphical results showing the variation in the output Stokes parameters for different input polarization of a beam which propagates along a birefringent fiber having small losses can be obtained. Figure (1) shows the change in output Stokes parameters for right circularly polarized input light as a function of the fiber length for a specific case in which circular birefringence and dichroism are both assumed absent in the fiber [11]. In this figure, the total intensity of the input light is seen to be dissipated by the fiber. This is due to attenuation by the fiber. Also, the output beam is observed to emerge elliptically polarized.

We now discuss specific cases of the Stokes-Mueller relation Eq.(15) and analyze some of the resulting polarization effects which emerge from different input polarization states of light propagating along a birefringent fiber. If the fiber is assumed to have negligible loss with small anisotropy along its length, then there is no dichroism and the linear birefringence relates to the anisotropy. In addition, we assume that there are external perturbations due to twists along the length of the fiber so that circular birefringence is present. Again, the corresponding Mueller matrix can be expanded in terms of the exponential of the birefringence vector and Lorentz generators to yield the following Stokes-Mueller relation

$$
\left(\begin{array}{c}
S_{0} \\
S_{1} \\
S_{2} \\
S_{3}
\end{array}\right)=\left(\begin{array}{cccc}
1 & 0 & 0 & 0 \\
0 & C_{\beta} & S_{2 \theta} S_{\beta} & -S_{2 \phi} C_{2 \theta} S_{\beta} \\
& +C_{2 \phi}^{2} C_{2 \theta}^{2}\left(1-C_{\beta}\right) & +C_{2 \theta}^{2} S_{2 \phi} C_{2 \varphi}\left(1-C_{\beta}\right) & +C_{2 \phi} C_{2 \theta} S_{2 \theta}\left(1-C_{\beta}\right) \\
0 & -S_{2 \theta} S_{\beta} & C_{\beta} & C_{2 \phi} C_{2 \theta} S_{\beta} \\
& +C_{2 \phi} C_{2 \theta}^{2} S_{2 \phi}\left(1-C_{\beta}\right) & +C_{2 \theta}^{2} S_{2 \phi}^{2}\left(1-C_{\beta}\right) & +S_{2 \phi} C_{2 \theta} S_{2 \theta}\left(1-C_{\beta}\right) \\
0 & S_{2 \phi} C_{2 \theta} S_{\beta} & -C_{2 \phi} C_{2 \theta} S_{\beta} & C_{\beta} \\
& +C_{2 \phi} C_{2 \theta} S_{2 \theta}\left(1-C_{\beta}\right) & +S_{2 \phi} C_{2 \theta} S_{2 \theta}\left(1-C_{\beta}\right) & +S_{2 \theta}^{2}\left(1-C_{\beta}\right)
\end{array}\right)\left(\begin{array}{c}
S_{00} \\
S_{10} \\
S_{20} \\
S_{30}
\end{array}\right)
$$

where $C_{2 \phi}=\cos 2 \phi, C_{2 \theta}=\cos 2 \theta, S_{2 \theta}=\sin 2 \theta, S_{\beta}=\sin \beta z$ and $C_{\beta}=\cos \beta z$.

Using Eq.(16), numerical results for the output Stokes parameters for different cases of input light (initially linearly polarized light, linear $+45^{\circ}$ polarized light, right circularly polarized light or elliptically polarized light) as a function of fiber length can be obtained. Figure (2) illustrates 
the variation in the output Stokes parameters as a function of fiber length for elliptically polarized ( $45^{\circ}$ azimuth and $22.5^{\circ}$ ellipticity) input light [11]. It is observed that for this input polarization and with circular birefringence present, the Stokes parameters vary sinusoidally with length. However, it is further observed that when loss effects are neglected and circular birefringence is assumed absent, the Stokes parameters remain constant for any input polarization. Thus, the presence of circular birefringence induces a variation in the polarization state of the propagating light in a lossless fiber. It is also observed that the beam emerging from the fiber has the same form as the input polarization for all cases of input polarization regardless of the presence or absence of circular birefringence. That is, for linearly horizontally polarized input light, the output light is observed to be linearly polarized on-axis and similarly for off-axis, circular and elliptical input polarizations.

\section{CONCLUSIONS}

In this paper, we have used the Lorentz group formalism to obtain analytical solutions to the evolution equations for the Stokes parameters. These solutions can be used to numerically obtain the polarization dynamics of a light beam along a birefringent single mode optical fiber subjected to a bend or twist. To test the validity of the solutions, we applied them to the test case of a straight fiber. The basic polarization effects of linear and circular birefringence and dichroism have been observed and presented. We have noted that when loss effects in a birefringent single mode optical fiber are neglected and circular birefringence is further assumed absent, the Stokes parameters remain constant for any input polarization. In addition, the presence of circular birefringence induces a variation in the polarization state of the propagating light in a lossless fiber. As expected, when losses are considered, the total intensity of the input light decays exponentially. The total intensity is dissipated due to attenuation by the fiber.

\section{ACKNOWLEDGEMENTS}

This work is supported by a grant from the Swedish International Cooperation Development Agency within the framework of the Associateship Scheme of the Abdus Salam International Centre for Theoretical Physics, Trieste, Italy. G. C. Ishiekwene acknowledges support of the International Atomic Energy Agency. 


\section{REFERENCES}

1. M. V. Tratnik and J. E. Sipe, "Nonlinear polarization dynamics I. The single-pulse equations, "Phys. Rev. A, 35 (7), 2965-2975, (1987).

2. R. Dandliker, "Rotational effects of polarization in optical fibers," in Anisotropic and Nonlinear Waveguides, C. G. Somenda and G. Stegeman, (Editors), Elsevier Science Publishers B. V., 39-76, (1992).

3. Y. P. Svirko and N. I. Zheludev, Polarization of Light in Nonlinear Optics, John Wiley and Sons, (1998).

4. K. L. Sala, "Nonlinear refractive-index phenomena in isotropic media subjected to a dc electric field: Exact solutions," Phys. Rev. A, 29 (4), 1944-1956, (1984).

5. G. Gregori and S. Wabnitz, "New eaxct solutions and bifurcations in the spatial distribution of polarization in third-order nonlinear optical interactions," Phys. Rev. Lett. 56 (6), 600-603, (1986).

6. B. Daino, G. Gregori, and S. Wabnitz, "New all-optical devices based on third-order nonlinearity of birefringent fibers," Opt. Lett. 11, 42-44, (1986).

7. R. F. Fox, "Gaussian stochastic processes in physics," Phys. Rep. 48, 179-283, (1978).

8. C. S. Brown and A. E. Bak, "Unified formalism for polarization optics with application to polarimetry on a twisted optical fiber," Opt. Eng. 34 (6), 1625-1635, (1995).

9. A. E. Bak, C. S. Brown, G. C. Ishiekwene, M. W. Shute Sr, "A review of the unified polarization calculus based on the lorentz group," unpublished.

10. A. E. Bak, F. K. A. Allotey, C. S. Brown, G. C. Ishiekwene, "The unified polarization calculus applied to analogous dynamical systems" unpublished.

11. G. C. Ishiekwene, "Nonlinear polarization effects in a birefringent single mode optical fiber," Ph.D. Thesis, (2000). 